\title{
Recurrence after endoscopic resection of small rectal neuroendocrine tumors: a retrospective cohort study
}

\author{
Sukit Pattarajierapan, Supakij Khomvilai \\ Surgical Endoscopy Colorectal Division, Department of Surgery, Faculty of Medicine, Chulalongkorn University, Bangkok, Thailand
}

Purpose: According to the European Neuroendocrine Tumor Society consensus guidelines, rectal neuroendocrine tumors (NETs) up to $10 \mathrm{~mm}$ in size and without poor prognostic factors could be safely removed with endoscopic resection, suggesting omitting surveillance colonoscopy after complete resection. However, the benefit of surveillance colonoscopy is still unknown. In this study, we aimed to report the outcomes after endoscopic resection of small rectal NETs using our surveillance protocol.

Methods: This retrospective cohort study included patients who underwent endoscopic resection for rectal NETs sized up to $10 \mathrm{~mm}$ from January 2013 to December 2019 at our center. We excluded patients without surveillance colonoscopy and those lost to follow-up. We strictly performed surveillance colonoscopy 1 year after endoscopic resection, and every 2 to 3 years thereafter. The primary outcomes were tumor recurrence and occurrence of metachronous tumors during followup.

Results: Of the 54 patients who underwent endoscopic resection for rectal NETs during the study period, 46 were enrolled in this study. The complete resection rates by endoscopic mucosal resection, precutting endoscopic mucosal resection, and endoscopic submucosal dissection were $92.3 \%$ (12 of 13), 100\% (21 of 21), and 100\% (12 of 12), respectively. There was no local or distant recurrence during the median follow-up of 39 months. However, we found that $8.7 \%$ (4 of 46 ) of patients developed metachronous NETs. All metachronous lesions were treated with precutting endoscopic mucosal resection.

Conclusion: Surveillance colonoscopy is reasonable after endoscopic resection of small rectal NETs for timely detection and treatment of metachronous lesions. However, larger collaborative studies are needed to influence the guidelines.

Keywords: Endoscopic mucosal resection; Endoscopic submucosal dissection; Neuroendocrine tumors; Rectum; Recurrence

\section{INTRODUCTION}

Neuroendocrine tumors (NETs) are rare neoplasms that originate from the Kulchitsky cells (i.e., enterochromaffin cells) located in the crypts of Lieberkuhn. Rectal NETs are known to

Received: Jan 6, 2021 - Revised: Feb 23, 2021 - Accepted: Feb 24, 2021

Correspondence to: Sukit Pattarajierapan, M.D.

Surgical Endoscopy Colorectal Division, Department of Surgery, Faculty of Medicine, Chulalongkorn University, 1873 Rama IV Road, Pathumwan, Bangkok 10330, Thailand

Tel: +66-2-256-4400, Fax: +66-2-256-4194

E-mail: sukit.p@chulahospital.org

ORCID: https://orcid.org/0000-0003-1042-8476

(C) 2022 The Korean Society of Coloproctology

This is an open-access article distributed under the terms of the Creative Commons Attribution NonCommercial License (https://creativecommons.org/licenses/by-nc/4.0) which permits unrestricted noncommercial use, distribution, and reproduction in any medium, provided the original work is properly cited. have the best prognosis of all NETs, with an $88.2 \%$ 5-year survival rate [1]. They represent $16 \%$ of all NETs and $27 \%$ of gastrointestinal NETs [2]. The Surveillance, Epidemiology, and End Results registry database of the National Cancer Institute shows that the age-adjusted incidence of rectal NETs has increased about tenfold over the last 35 years [3]. The rapid increase in incidence might be associated with the increased use of colorectal cancer screening [4]. Screening colonoscopy leads to the detection of rectal NETs of smaller size and earlier stage; $93 \%$ to $100 \%$ of rectal NETs detected with screening colonoscopy are less than $10 \mathrm{~mm}$ in size $[1,5]$.

According to the European Neuroendocrine Tumor Society (ENETS) consensus guidelines for the management of colorectal NETs, rectal NETs up to $10 \mathrm{~mm}$ in size and without poor prognostic factors (G3, lymphovascular invasion [LVI], and muscularis propria invasion) could be safely removed with endoscopic re- 
section because of the low risk of metastasis $(2 \%)[6,7]$. Endoscopic mucosal resection (EMR), precutting EMR (P-EMR), and endoscopic submucosal dissection (ESD) were reported as effective endoscopic treatment [8-13]. Endoscopists usually select the resection technique based on their preference.

Tumor recurrence after endoscopic resection for rectal NETs of up to $10 \mathrm{~mm}$ is uncommon $(0 \%-4.2 \%)$ [14-17]. Moreover, the 5 -year overall survival was reported to be $95 \%$ to $100 \%[5,18,19]$. The ENETS guidelines suggest omitting surveillance colonoscopy after complete resection of rectal NETs less than $10 \mathrm{~mm}$ in size [7]. Similarly, the National Comprehensive Cancer Network (NCCN) guidelines for NETs of the gastrointestinal tract state that surveillance colonoscopy is not required after resection of rectal NETs smaller than $10 \mathrm{~mm}$ [20]. In previous studies with the long-term outcome of rectal NET resection, not all patients underwent regular surveillance colonoscopy [10, 21-24].

In this study, we report the outcome of endoscopic resection for rectal NETs up to $10 \mathrm{~mm}$ in size using our surveillance protocol, with focus on the tumor recurrence and occurrence of metachronous tumors. According to our surveillance protocol, surveillance colonoscopy is performed 1 year after endoscopic resection and every 2 to 3 years thereafter. Additionally, patients with high-risk features (NETs grade of $\geq 2$ and size of $\geq 10 \mathrm{~mm}$ ) undergo annual surveillance abdominal computed tomography (CT).

\section{METHODS}

\section{Study design and population}

Patients who underwent endoscopic resection for rectal NETs at the Surgical Endoscopy Colorectal Division of King Chulalongkorn Memorial Hospital in Bangkok, Thailand from January 1, 2013 to December 31, 2019 were recruited prospectively within the electronic database. We retrospectively analyzed the data from this cohort, including patients' characteristics, endoscopic characteristics, pathologic reports, and follow-up information. Patients who did not undergo surveillance colonoscopy and those who were lost to follow-up were excluded from the analysis.

Due to the retrospective design of the study, consent was waived by the ethics committee for the entire study. The study protocol was approved by the Institutional Review Board of Chulalongkorn University (No. 196-63).

\section{Endoscopic resection}

Endoscopic resection for rectal NETs sized up to $10 \mathrm{~mm}$ was usually performed during the initial colonoscopy by the attending staff of the colorectal unit. Abdominal CT was performed after resection because the risk for metastasis for these tumors is very low. Because endoscopic ultrasound is not available in our unit, we selected the endoscopic resection technique according to the tumor size and endoscopic morphology (Fig. 1).

For EMR, after submucosal saline injection (NeedleMaster injection needle, Olympus Corp., Tokyo, Japan), a 10-mm snare
(SnareMaster, Olympus Corp.) was used for resection. Finally, we performed endoscopic clipping (EZ clip, Olympus Corp.) in all cases.

For P-EMR, we performed a circumferential incision to the submucosa around the lesion using an endoscopic knife (Dual Knife, Olympus Corp.) before snare resection.

ESD was primarily performed using the Dual Knife. We used glycerol for submucosal lifting. A transparent distal cap was used from the start of ESD to provide countertraction for dissection.

\section{Pathological evaluation}

Complete resection was defined as an en bloc resection without lateral or vertical margin involvement on pathological assessment. All resected specimens were examined with H\&E and immunohistochemical staining with synaptophysin and chromogranin. The mitotic count and Ki-67 index were measured. LVI was evaluated. Grading of rectal NETs was performed according to the 2010 World Health Organization (WHO) classification [25].

\section{Outcomes and definitions}

The primary outcome was tumor recurrence, including local and distant recurrence and the occurrence of metachronous NETs. Secondary outcomes included complete resection rate, procedural time, and complications.

Local recurrence was defined as development of NETs adjacent to the previous scar after endoscopic resection. Distant recurrence was defined as development of NETs beyond the rectal wall. Synchronous rectal NETs were defined as more than 1 rectal NETs in a patient detected on colonoscopy up to 6 months after diagnosis. Metachronous rectal NETs were defined as NETs detected at a site distant from the primary lesion more than 6 months after initial diagnosis. For the evaluation of metachronous lesions, a cutoff period of 6 months after initial diagnosis, was used to exclude initial tumors that had missed or local recurrence from incomplete excision.

\section{Follow-up protocol}

We used our own surveillance protocol because there is no wellestablished surveillance strategy. All patients underwent surveillance colonoscopy 1 year after endoscopic resection and every 2 to 3 years thereafter. Additionally, patients with high-risk features (NETs grade of $\geq 2$ and size of $\geq 10 \mathrm{~mm}$ ) underwent annual surveillance abdominal CT.

\section{Statistical analysis}

The data were analyzed using Stata ver. 15.1 (Stata Corp., College Station, TX, USA). Continuous variables were assessed by plotting histograms to assess whether data were normally distributed. They were compared using one-way analysis of variance, followed by Bonferroni post hoc comparisons, Kruskal-Wallis rank test, and Wilcoxon rank-sum test as appropriate. Categorical variables were compared using two-tailed chi-square tests or Fisher exact 

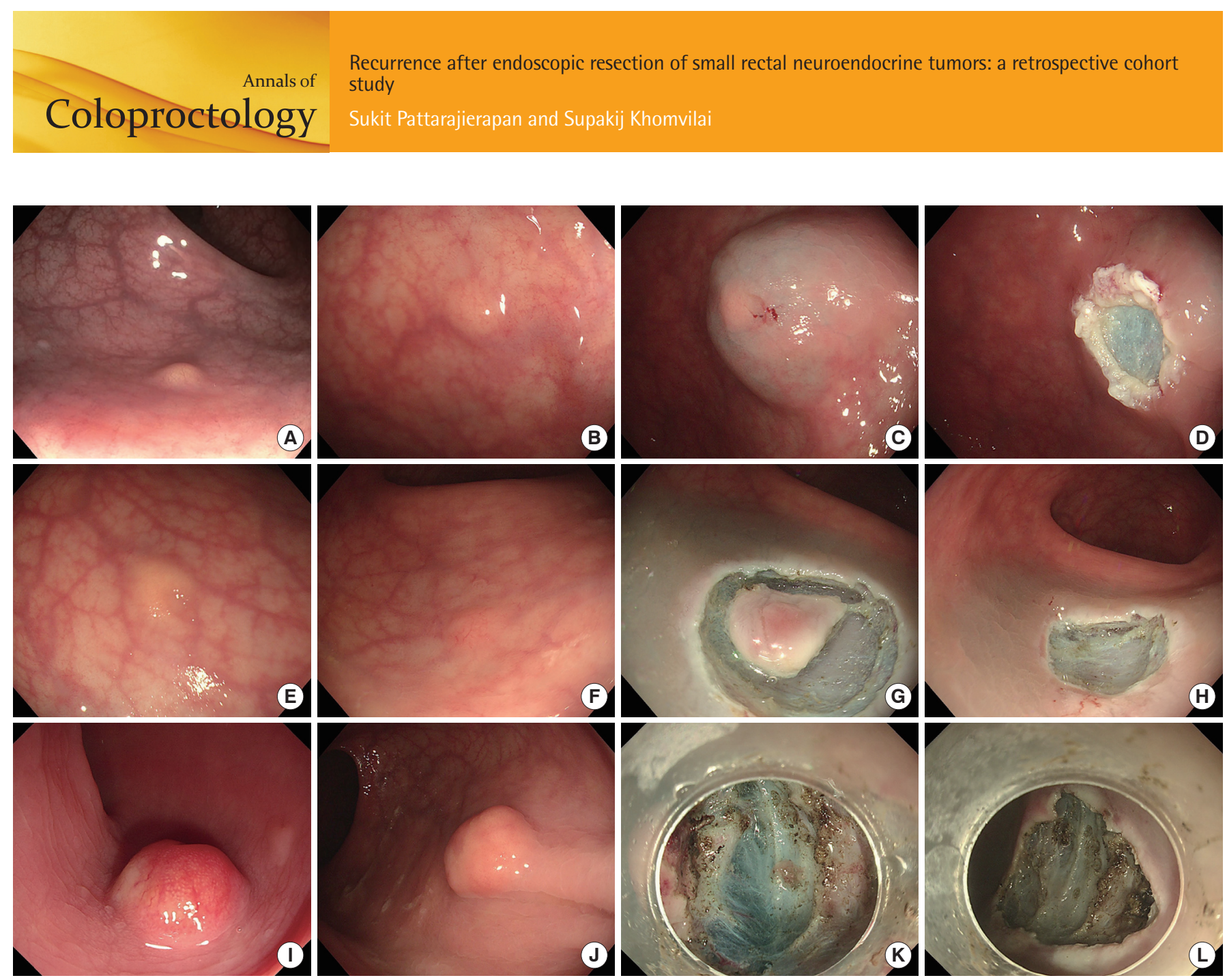

Fig. 1. Criteria for selection of an endoscopic resection technique in our institution. (A-D) Endoscopic mucosal resection (EMR), (E-H) precutting EMR (P-EMR), and (I-L) endoscopic submucosal dissection (ESD). (A, B) Rectal neuroendocrine tumors (NETs) of $\leq 5$ mm in the superficial submucosa. (C) Lifting. (D) Wound after snaring. (E, F) Rectal NETs of $\leq 5 \mathrm{~mm}$ in the deep submucosa. (G) Circumferential incision. (H) Wound after P-EMR. (I, J) Rectal NETs 6 to $10 \mathrm{~mm}$. (K) Submucosal dissection. (L) Wound after ESD.

test. A P-value of $<0.05$ was considered to be statistically significant.

\section{RESULTS}

During the study period, 54 patients underwent endoscopic resection for rectal NETs. Three patients were lost to follow-up, and 5 patients did not undergo surveillance colonoscopy. Therefore, 46 patients were included in this study (Fig. 2).

Table 1 shows the patients' characteristics. EMR, P-EMR, and ESD were performed in 13,21 , and 12 patients, respectively. The overall mean age of the patients was 59 years (range, $38-78$ years). There was a significant difference among the groups regarding the tumor size $(\mathrm{P}=0.003)$; tumors in the ESD group were significantly larger than those in the EMR and P-EMR groups $(\mathrm{P}=0.007$ and $\mathrm{P}=0.009$, respectively). The follow-up period in the EMR group was longer than that in the P-EMR and ESD groups $(\mathrm{P}<0.001$ and $\mathrm{P}=0.033$, respectively). Only 1 patient $(2.2 \%, 1$ of 46) had multiple synchronous rectal NETs on initial colonoscopy.
After pathologic evaluation, only 1 patient in the EMR group had a grade 2 NET based on the WHO classification; the remaining patients had grade 1 NETs. None of the patients had LVI or regional lymph node or distant metastasis on abdominal CT.

The clinical outcomes in each endoscopic resection modality are summarized in Table 2. The complete resection rate was $100 \%$ in the P-EMR and ESD groups, and $92.3 \%$ (12 of 13) in the EMR group. The deep margin of one patient in the EMR group could not be assessed due to the cauterized effect. After discussion with the patient, we decided to do a close follow-up; there was no recurrence during a follow-up of 86 months. The procedural time was longer in the ESD group than that in the EMR and P-EMR groups $(\mathrm{P}<0.001$ and $\mathrm{P}=0.013$, respectively), and the procedural time of the P-EMR group was longer than that of the EMR group $(\mathrm{P}<0.001)$. No complication occurred in any of the groups.

Table 3 shows the tumor recurrence and occurrence of metachronous tumor in each endoscopic resection modality. After endoscopic resection, there was no local or distant recurrence during the median follow-up of 39 months (range, 12-86 months). 


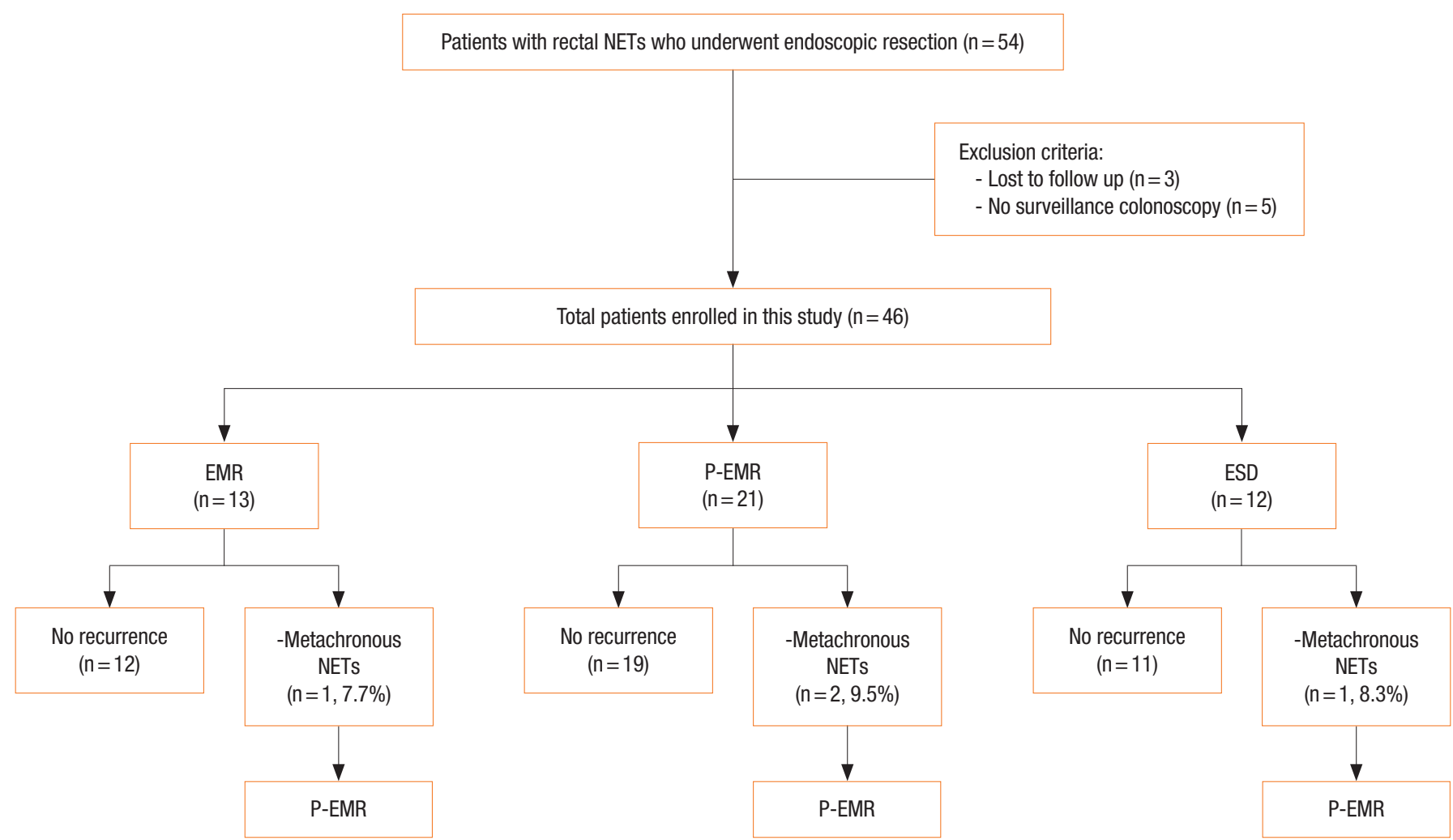

Fig. 2. Flowchart of enrolled patients and recurrence after endoscopic resection for rectal neuroendocrine tumors (NETs). EMR, endoscopic mucosal resection; P-EMR, precutting EMR; ESD, endoscopic submucosal dissection.

Table 1. Baseline characteristics of the study group

\begin{tabular}{|c|c|c|c|c|c|c|c|}
\hline \multirow{2}{*}{ Variable } & \multirow{2}{*}{$\begin{array}{c}\text { EMR } \\
(n=13)\end{array}$} & \multirow{2}{*}{$\begin{array}{l}\text { P-EMR } \\
(n=21)\end{array}$} & \multirow{2}{*}{$\begin{array}{c}\text { ESD } \\
(n=12)\end{array}$} & \multicolumn{4}{|c|}{ P-value } \\
\hline & & & & Total & EMR vs. P-EMR & EMR vs. ESD & P-EMR vs. ESD \\
\hline Age (yr) & $57 \pm 8$ & $60 \pm 9$ & $61 \pm 12$ & 0.596 & 1 & 0.947 & 1 \\
\hline Sex, male:female & $6: 7$ & $13: 8$ & $10: 2$ & & & & \\
\hline Endoscopically estimated size (mm) & $5.8 \pm 2.7$ & $6.2 \pm 2.3$ & $9.2 \pm 2.8$ & $0.003^{*}$ & 1 & $0.007^{*}$ & $0.009^{*}$ \\
\hline Single lesion & $12(92.3)$ & $21(100)$ & $12(100)$ & & & & \\
\hline Multiple lesions & $1(7.7)$ & & & & & & \\
\hline \multicolumn{8}{|l|}{ WHO grading } \\
\hline Grade 1 & $12(92.3)$ & $21(100)$ & $12(100)$ & & & & \\
\hline Grade 2 & $1(7.7)$ & $0(0)$ & $0(0)$ & & & & \\
\hline Grade 3 & $0(0)$ & $0(0)$ & $0(0)$ & & & & \\
\hline Lymphovascular invasion & $0(0)$ & $0(0)$ & $0(0)$ & & & & \\
\hline \multicolumn{8}{|l|}{ Ki-67 index (\%) } \\
\hline$<3$ & 12 (92.3) & $21(100)$ & $12(100)$ & & & & \\
\hline $3-20$ & $1(7.7)$ & & & & & & \\
\hline \multicolumn{8}{|l|}{$>20$} \\
\hline Follow-up (mo) & $55(42-84)$ & $27(18-42)$ & $39(21-50)$ & $<0.001^{*}$ & $<0.001^{*}$ & $0.033^{*}$ & 0.244 \\
\hline
\end{tabular}

Values are presented as mean \pm standard deviation, number only, number (\%), or median (interquartile range).

EMR, endoscopic mucosal resection; P-EMR, precutting EMR; ESD, endoscopic submucosal dissection; WHO, World Health Organization.

${ }^{*} \mathrm{P}<0.05$. 


\section{Coloproctology}

Table 2. Clinical outcomes in each endoscopic resection modality

\begin{tabular}{lccccc}
\hline Variable & $\begin{array}{c}\text { EMR } \\
(\mathrm{n}=13)\end{array}$ & $\begin{array}{c}\text { P-EMR } \\
(\mathrm{n}=21)\end{array}$ & $\begin{array}{c}\text { ESD } \\
(\mathrm{n}=12)\end{array}$ & Total & EMR vs. P-EMR EMR vs. ESD P-EMR vs. ESD \\
\hline Complete resection rate & $12(92.3)$ & $21(100)$ & $12(100)$ & 0.543 & \\
$\begin{array}{l}\text { Pathological margin involvement } \\
\text { Lateral }\end{array}$ & 0 & 0 & 0 & & \\
Deep & $1(7.7)$ & 0 & 0 & & $<0.001^{*}$ \\
Procedural time (min) & $4(3-4)$ & $11(8-15)$ & $18(12-29)$ & $<0.001^{*}$ & $<0.001^{*}$ \\
Complication (delayed bleeding, perforation) & 0 & 0 & 0 & & $0.013^{*}$ \\
\hline
\end{tabular}

Values are presented as number (\%) or median (interquartile range).

EMR, endoscopic mucosal resection; P-EMR, precutting EMR; ESD, endoscopic submucosal dissection.

${ }^{*} \mathrm{P}<0.05$.

Table 3. Tumor recurrence and occurrence of metachronous tumor in each endoscopic resection modality

\begin{tabular}{lcccc}
\hline & EMR $(\mathrm{n}=13)$ & P-EMR $(\mathrm{n}=21)$ & ESD $(\mathrm{n}=12)$ & Total $(\mathrm{n}=46)$ \\
\hline Local recurrence & $0(0)$ & $0(0)$ & $0(0)$ & $0(0)$ \\
Distant recurrence & $0(0)$ & $0(0)$ & $0(0)$ & $0(0)$ \\
Metachronous NETS & $1(7.7)$ & $2(9.5)$ & $1(8.3)$ & $4(8.7)$ \\
Time to metachronous NETS $(\mathrm{mo})$ & 47 & $31.5(19-44)$ & 39 & $41.5(19-47)$ \\
\hline
\end{tabular}

Values are presented as number (\%), median only, or median (range).

EMR, endoscopic mucosal resection; P-EMR, precutting EMR; ESD, endoscopic submucosal dissection; NET, neuroendocrine tumor.

Table 4. Characteristics of patients with metachronous NETs

\begin{tabular}{|c|c|c|c|c|c|c|c|c|c|c|}
\hline \multirow[b]{2}{*}{ No. } & \multicolumn{5}{|c|}{ Primary NETs } & \multicolumn{4}{|c|}{ Metachronous NETs } & \multirow[b]{2}{*}{ Endoscopist } \\
\hline & $\begin{array}{l}\text { Size } \\
(\mathrm{mm})\end{array}$ & Location & $\begin{array}{c}\text { Resection } \\
\text { modality }\end{array}$ & Grade & $\begin{array}{c}\text { Synchronous } \\
\text { NETs }\end{array}$ & $\begin{array}{c}\text { Time to } \\
\text { metachronous } \\
\text { lesion (mo) }\end{array}$ & $\begin{array}{l}\text { Size } \\
(\mathrm{mm})\end{array}$ & Location & $\begin{array}{c}\text { Resection } \\
\text { modality }\end{array}$ & \\
\hline 1 & 5 & Lower rectum & EMR & 1 & No & 47 & 7 & Middle rectum & P-EMR & S.K. \\
\hline 2 & 10 & Middle rectum & ESD & 1 & No & 39 & 3 & Middle rectum & P-EMR & S.K. \\
\hline 3 & 6 & Middle rectum & P-EMR & 1 & No & 44 & 3 & Lower rectum & P-EMR & S.K. \\
\hline 4 & 4 & Middle rectum & P-EMR & 1 & No & 19 & 3 & Lower rectum & P-EMR & S.K. \\
\hline
\end{tabular}

NET, neuroendocrine tumor; EMR, endoscopic mucosal resection; P-EMR, precutting EMR; ESD, endoscopic submucosal dissection.

However, we found that $8.7 \%$ ( 4 of 46 ) of patients developed metachronous rectal NETs. The median time to metachronous NETs was 41.5 months (range, 19-47 months). The mean diameter of the metachronous lesions was $4 \mathrm{~mm}$ (range, 3-7 mm). All metachronous NETs were resected with P-EMR and classified as grade 1 NETs. The characteristics of all patients with metachronous NETs are described in more detail in Table 4.

\section{DISCUSSION}

In this study, we analyzed the outcomes of endoscopic resection for rectal NETs up to $10 \mathrm{~mm}$ in size using our surveillance protocol based on surveillance colonoscopy performed 1 year after endoscopic resection and every 2 to 3 years thereafter. During the median follow-up period of 39 months, there was no local or distant tumor recurrence; however, $8.7 \%$ of the patients developed metachronous rectal NETs.

Previous studies showed that the recurrence rate after endoscopic resection for rectal NETs was low (0\%-4.2\%) [14-17, 21, $24,26-28]$. There was a diminutively increased chance of local recurrence with a positive resection margin. In a multicenter study, Moon et al. [29] found that local recurrence occurred in $0.74 \%$ of 407 patients who underwent endoscopic resection. They had $18.7 \%$ positive and $17.7 \%$ indeterminate margins. While Chung et al. [30] reported that no local recurrence occurred with 3.9\% positive resection margins. Similarly, our results showed no local recurrence with $2.2 \%$ positive resection margins.

Few studies have reported metachronous NETs on surveillance 
colonoscopy. Moon et al. [29] found that $0.74 \%$ of patients developed metachronous NETs during a median follow-up of 45 months. Kwak et al. [24] detected only 1 case (1\%) of metachronous NETs during 84 months of follow-up. Recently, Chung et al. [30] reported that 9 of 329 patients (2.7\%) with rectal NETs of up to $1 \mathrm{~cm}$ developed metachronous NETs after endoscopic resection. Our study had a much higher rate $(8.7 \%)$ of metachronous rectal NETs compared with those studies. The pathogenesis of metachronous NETs is still unknown. Chung et al. [30] found an association between development of metachronous lesions and the presence of synchronous lesions at initial diagnosis (hazard ratio, 1.75; $\mathrm{P}<0.001)$. However, in our study, only 1 patient had synchronous lesions, and this patient did not develop a metachronous lesion during the follow-up period.

The NCCN guidelines for NETs of the gastrointestinal tract state that surveillance colonoscopy is not required after resection of rectal NETs less than $10 \mathrm{~mm}$ in size [20]. Similarly, the ENETS consensus guidelines for colorectal neuroendocrine neoplasms do not recommend surveillance colonoscopy for rectal NETs sized less than $10 \mathrm{~mm}$ treated with complete resection [7]. Although these guidelines do not recommend it, many endoscopists still perform surveillance colonoscopy. The results of our study indicate the potential benefit of surveillance colonoscopy. We detected $8.7 \%$ of metachronous NETs during the 39-month median follow-up. The mean diameter of metachronous NETs was $4 \mathrm{~mm}$, and we resected them with P-EMR. If surveillance colonoscopy was not performed, they could progress to larger NETs requiring radical surgery. However, the cost-effectiveness and the interval of surveillance colonoscopy are still unknown. Additional larger studies are needed to address the role of surveillance colonoscopy after endoscopic resection of rectal NETs.

Rectal NETs less than $10 \mathrm{~mm}$ in size with no muscularis propria invasion or lymph node metastasis are the indication for endoscopic resection $[7,20]$. However, there is no specific recommendation on the optimal endoscopic resection technique for rectal NETs. Endoscopists usually select the resection technique based on their preference. Among the available techniques, EMR is simple and quick, but it has the lowest pathological complete resection rate (28.6\%-82.1\%) [8-11]. P-EMR is a modification of EMR and has a better pathological complete resection rate $(81.2 \%-$ 96.7\%) $[9,12,13,26,27]$. Although ESD has a pathological complete resection rate of $77.8 \%$ to $100 \%$, it is technically difficult and has a higher rate of complications [8, 10, 12, 21, 26, 27]. In our study, we selected the endoscopic treatment modality based on the tumor size and endoscopic morphology due to the unavailability of endoscopic ultrasound. If the tumors were less than 5 $\mathrm{mm}$ and located in the deep submucosa, we selected P-EMR. For tumors located in the superficial submucosa, we performed EMR. ESD was reserved for NETs sized $6 \mathrm{~mm}$ or more. The pathological complete resection rates of EMR, P-EMR, and ESD in this study were $92.3 \%, 100 \%$, and $100 \%$, respectively, and there were no complications. Therefore, we believe that the tumor size and endoscopic morphology could be considered to select the endoscopic treatment modality when endoscopic ultrasound is not available.

This study has several limitations. First, $14.8 \%$ of patients (8 of 54) were excluded from the study because they had not undergone surveillance colonoscopy or were lost to follow-up. The rate of metachronous NETs might have been lower if a higher rate of patients adhered to the surveillance protocol. Second, the number of patients was small. We had only one patient with synchronous rectal NETs; thus, an association analysis between synchronous and metachronous rectal NETs was impossible. Third, 4 endoscopists performed endoscopic resection in this study. We cannot avoid the variation of competency in endoscopic evaluation and treatment among our staff. However, most procedures (37 of 46, $80.4 \%$ ) were performed by S.K., who has performed $>10,000$ colonoscopies and $>400$ ESD procedures. Fourth, the metachronous NETs could be the overlooked lesions on the initial colonoscopies because they are small with 4-mm mean diameter, which is difficult to prove. However, the initial colonoscopies of all the patients with metachronous NETs were performed by S.K., who is the most experienced endoscopist in this center. Finally, the median follow-up time was 39 months in this study. Indeed, studies with a longer follow-up duration are needed to address the incidence of metachronous rectal NETs.

In conclusion, endoscopic resection is effective for rectal NETs up to $10 \mathrm{~mm}$ in size. Despite the absence of local or distant recurrence in this study, the incidence of metachronous rectal NETs was higher than that reported in previous studies. Therefore, surveillance colonoscopy is reasonable after endoscopic resection for timely detection and treatment of metachronous rectal NETs. However, larger collaborative studies are required to influence the guidelines.

\section{CONFLICT OF INTEREST}

No potential conflict of interest relevant to this article was reported.

\section{FUNDING}

None.

\section{REFERENCES}

1. Scherübl H. Rectal carcinoids are on the rise: early detection by screening endoscopy. Endoscopy 2009;41:162-5.

2. Jetmore AB, Ray JE, Gathright JB Jr, McMullen KM, Hicks TC, Timmcke AE. Rectal carcinoids: the most frequent carcinoid tumor. Dis Colon Rectum 1992;35:717-25.

3. Tsikitis VL, Wertheim BC, Guerrero MA. Trends of incidence and survival of gastrointestinal neuroendocrine tumors in the United States: a seer analysis. J Cancer 2012;3:292-302. 
4. Dasari A, Shen C, Halperin D, Zhao B, Zhou S, Xu Y, et al. Trends in the incidence, prevalence, and survival outcomes in patients with neuroendocrine tumors in the United States. JAMA Oncol 2017; 3:1335-42.

5. Bertani E, Ravizza D, Milione M, Massironi S, Grana CM, Zerini D, et al. Neuroendocrine neoplasms of rectum: a management update. Cancer Treat Rev 2018;66:45-55.

6. McDermott FD, Heeney A, Courtney D, Mohan H, Winter D. Rectal carcinoids: a systematic review. Surg Endosc 2014;28:20206.

7. Ramage JK, De Herder WW, Delle Fave G, Ferolla P, Ferone D, Ito $\mathrm{T}$, et al. ENETS consensus guidelines update for colorectal neuroendocrine neoplasms. Neuroendocrinology 2016;103:139-43.

8. Kim KM, Eo SJ, Shim SG, Choi JH, Min BH, Lee JH, et al. Treatment outcomes according to endoscopic treatment modalities for rectal carcinoid tumors. Clin Res Hepatol Gastroenterol 2013;37: 275-82.

9. Huang J, Lu ZS, Yang YS, Yuan J, Wang XD, Meng JY, et al. Endoscopic mucosal resection with circumferential incision for treatment of rectal carcinoid tumours. World J Surg Oncol 2014;12:23.

10. Park HW, Byeon JS, Park YS, Yang DH, Yoon SM, Kim KJ, et al. Endoscopic submucosal dissection for treatment of rectal carcinoid tumors. Gastrointest Endosc 2010;72:143-9.

11. Choi HH, Kim JS, Cheung DY, Cho YS. Which endoscopic treatment is the best for small rectal carcinoid tumors? World J Gastrointest Endosc 2013;5:487-94.

12. Cheung DY, Choi SK, Kim HK, Kim SS, Chae HS, Seo KJ, et al. Circumferential submucosal incision prior to endoscopic mucosal resection provides comparable clinical outcomes to submucosal dissection for well-differentiated neuroendocrine tumors of the rectum. Surg Endosc 2015;29:1500-5.

13. So H, Yoo SH, Han S, Kim GU, Seo M, Hwang SW, et al. Efficacy of precut endoscopic mucosal resection for treatment of rectal neuroendocrine tumors. Clin Endosc 2017;50:585-91.

14. Kwaan MR, Goldberg JE, Bleday R. Rectal carcinoid tumors: review of results after endoscopic and surgical therapy. Arch Surg 2008;143: 471-5.

15. He L, Deng T, Luo H. Efficacy and safety of endoscopic resection therapies for rectal carcinoid tumors: a meta-analysis. Yonsei Med J 2015;56:72-81.

16. Wei R, Lo OS, Law WL. Surgical management and outcome of rectal carcinoids in a university hospital. World J Surg Oncol 2015; 13:31.

17. Kim J, Kim JH, Lee JY, Chun J, Im JP, Kim JS. Clinical outcomes of endoscopic mucosal resection for rectal neuroendocrine tumor. BMC Gastroenterol 2018;18:77.

18. Park CH, Cheon JH, Kim JO, Shin JE, Jang BI, Shin SJ, et al. Criteria for decision making after endoscopic resection of well-differentiated rectal carcinoids with regard to potential lymphatic spread. Endoscopy 2011;43:790-5.
19. Gleeson FC, Levy MJ, Dozois EJ, Larson DW, Wong Kee Song LM, Boardman LA. Endoscopically identified well-differentiated rectal carcinoid tumors: impact of tumor size on the natural history and outcomes. Gastrointest Endosc 2014;80:144-51.

20. National Comprehensive Cancer Network (NCCN). Neuroendocrine and adrenal tumors (version 2.2020) [Internet]. Plymouth Meeting (PA): NCCN: 2020 [cited 2021 Feb 23]. Available from: https://www.nccn.org/professionals/physician_gls/pdf/neuroendocrine.pdf.

21. Onozato Y, Kakizaki S, Iizuka H, Sohara N, Mori M, Itoh H. Endoscopic treatment of rectal carcinoid tumors. Dis Colon Rectum 2010;53:169-76.

22. Kim GU, Kim KJ, Hong SM, Yu ES, Yang DH, Jung KW, et al. Clinical outcomes of rectal neuroendocrine tumors $\leq 10 \mathrm{~mm}$ following endoscopic resection. Endoscopy 2013;45:1018-23.

23. Cha JH, Jung DH, Kim JH, Youn YH, Park H, Park JJ, et al. Longterm outcomes according to additional treatments after endoscopic resection for rectal small neuroendocrine tumors. Sci Rep 2019;9:4911.

24. Kwak MS, Chung SJ, Yang JI, Im JP, Park MJ, Lee C, et al. Longterm outcome of small, incidentally detected rectal neuroendocrine tumors removed by simple excisional biopsy compared with the advanced endoscopic resection during screening colonoscopy. Dis Colon Rectum 2018;61:338-46.

25. Bosman FT, Carneiro F, Hruban RH, Theise ND. WHO classification of tumours of the digestive system. 4 th ed. Lyon: International Agency for Research on Cancer; 2010.

26. Chen R, Liu X, Sun S, Wang S, Ge N, Wang G, et al. Comparison of endoscopic mucosal resection with circumferential incision and endoscopic submucosal dissection for rectal carcinoid tumor. Surg Laparosc Endosc Percutan Tech 2016;26:e56-61.

27. Wang XY, Chai NL, Linghu EQ, Li HK, Zhai YQ, Feng XX, et al. Efficacy and safety of hybrid endoscopic submucosal dissection compared with endoscopic submucosal dissection for rectal neuroendocrine tumors and risk factors associated with incomplete endoscopic resection. Ann Transl Med 2020;8:368.

28. Sung HY, Kim SW, Kang WK, Kim SY, Jung CK, Cho YK, et al. Long-term prognosis of an endoscopically treated rectal neuroendocrine tumor: 10-year experience in a single institution. Eur J Gastroenterol Hepatol 2012;24:978-83.

29. Moon CM, Huh KC, Jung SA, Park DI, Kim WH, Jung HM, et al. Long-term clinical outcomes of rectal neuroendocrine tumors according to the pathologic status after initial endoscopic resection: a KASID multicenter study. Am J Gastroenterol 2016;111: 1276-85.

30. Chung HG, Goh MJ, Kim ER, Hong SN, Kim TJ, Chang DK, et al. Recurrence pattern and surveillance strategy for rectal neuroendocrine tumors after endoscopic resection. J Gastroenterol Hepatol 2021;36:968-73. 